\title{
Research Article \\ Coupled Coincidence Point and Coupled Common Fixed Point Theorems in Partially Ordered Metric Spaces with $w$-Distance
}

\author{
Mujahid Abbas, ${ }^{1}$ Dejan Ilić, ${ }^{2}$ and Muhammad Ali Khan ${ }^{1}$ \\ ${ }^{1}$ Department of Mathematics, Lahore University of Management Sciences, 54792 Lahore, Pakistan \\ ${ }^{2}$ Department of Mathematics, Faculty of Sciences and Mathematics, University of Niŝ, Viŝegradska 33, \\ 18000 Niŝ, Serbia
}

Correspondence should be addressed to Dejan Ilić, ilicde@ptt.rs

Received 7 April 2010; Accepted 18 October 2010

Academic Editor: Hichem Ben-El-Mechaiekh

Copyright (C) 2010 Mujahid Abbas et al. This is an open access article distributed under the Creative Commons Attribution License, which permits unrestricted use, distribution, and reproduction in any medium, provided the original work is properly cited.

\begin{abstract}
We introduce the concept of a $w$-compatible mapping to obtain a coupled coincidence point and a coupled point of coincidence for nonlinear contractive mappings in partially ordered metric spaces equipped with $w$-distances. Related coupled common fixed point theorems for such mappings are also proved. Our results generalize, extend, and unify several well-known comparable results in the literature.
\end{abstract}

\section{Introduction and Preliminaries}

In 1996, Kada et al. [1] introduced the notion of $w$-distance. They elaborated, with the help of examples, that the concept of $w$-distance is general than that of metric on a nonempty set. They also proved a generalization of Caristi fixed point theorem employing the definition of $w$-distance on a complete metric space. Recently, Ilić and Rakočević [2] obtained fixed point and common fixed point theorems in terms of $w$-distance on complete metric spaces (see also [3-9]).

Definition 1.1. Let $(X, d)$ be a metric space. A mapping $p: X \times X \rightarrow[0, \infty)$ is called a $w$ distance on $X$ if the following are satisfied:

$$
\left(\mathrm{w}_{1}\right) p(x, z) \leq p(x, y)+p(y, z) \text { for all } x, y, z \in X \text {, }
$$

$\left(\mathrm{w}_{2}\right)$ for any $x \in X, p(x, \cdot): X \rightarrow[0, \infty)$ is lower semicontinuous, 
$\left(\mathrm{w}_{3}\right)$ for any $\varepsilon>0$ there exists $\delta(\varepsilon)>0$ such that $p(z, x) \leq \delta$ and $p(z, y) \leq \delta$ imply $p(x, y) \leq \varepsilon$, for any $x, y, z \in X$.

The metric $d$ is a $w$-distance on $X$. For more examples of $w$-distances, we refer to [10].

Definition 1.2. Let $X$ be a nonempty set with a $w$-distance on $X$. Ones denotes the $w$-closure

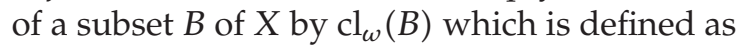

$$
\mathrm{cl}_{\omega}(B)=\left\{x \in X: p\left(x_{n}, x\right) \longrightarrow 0 \text { for some sequence }\left\{x_{n}\right\} \text { in } B\right\} \cup B
$$

The next Lemma is crucial in the proof of our results.

Lemma 1.3 (see [1]). Let $(X, d)$ be a metric space, and let $p$ be a w-distance on $X$. Let $\left\{x_{n}\right\}$ and $\left\{y_{n}\right\}$ be sequences in $X$, let $\alpha_{n}$ and $\beta_{n}$ be sequences in $[0, \infty)$ converging to 0 , and let $x, y, z \in X$. Then the following hold.

(1) If $p\left(x_{n}, y\right) \leq \alpha_{n}$ and $p\left(x_{n}, z\right) \leq \beta_{n}$ for any $n \in N$, then $y=z$. In particular, if $p(x, y)=$ $0, p(x, z)=0$ then $y=z$.

(2) If $p\left(x_{n}, y_{n}\right) \leq \alpha_{n}$ and $p\left(x_{n}, z\right) \leq \beta_{n}$ for any $n \in N$, then $y_{n}$ converges to $z$.

(3) If $p\left(x_{n}, x_{m}\right) \leq \alpha_{n}$ for any $m, n \in N$ with $n \prec m$, then $x_{n}$ is a Cauchy sequence.

(4) If $p\left(y, x_{n}\right) \leq \alpha_{n}$ for any $n \in N$, then $x_{n}$ is a Cauchy sequence.

Bhaskar and Lakshmikantham in [11] introduced the concept of coupled fixed point of a mapping $F: X \times X \rightarrow X$ and investigated some coupled fixed point theorems in partially ordered sets. They also discussed an application of their result by investigating the existence and uniqueness of solution for a periodic boundary value problem. Sabetghadam et al. in [12] introduced this concept in cone metric spaces. They investigated some coupled fixed point theorems in cone metric spaces. Recently, Lakshmikantham and Cirić [13] proved coupled coincidence and coupled common fixed point theorems for nonlinear contractive mappings in partially ordered complete metric spaces which extend the coupled fixed point theorem given in [11]. The following are some other definitions needed in the sequel.

Definition 1.4 (see [12]). Let $X$ be any nonempty set. Let $F: X \times X \rightarrow X$ and $g: X \rightarrow X$ be two mappings. An ordered pair $(x, y) \in X \times X$ is called

(1) a coupled fixed point of a mapping $F: X \times X \rightarrow X$ if $x=F(x, y)$ and $y=F(y, x)$,

(2) a coupled coincidence point of hybrid pair $\{F, g\}$ if $g(x)=F(x, y)$ and $g(y)=$ $F(y, x)$ and $(g x, g y)$ is called coupled point of coincidence,

(3) a common coupled fixed point of hybrid pair $\{F, g\}$ if $x=g(x)=F(x, y)$ and $y=g(y)=F(y, x)$.

Note that if $(x, y)$ is a coupled fixed point of $F$, then $(y, x)$ is also a coupled fixed point of the mapping $F$.

Definition 1.5. Let $X$ be any nonempty set. Mappings $F: X \times X \rightarrow X$ and $g: X \rightarrow X$ are called $w$-compatible if $g(F(x, y))=F(g x, g y)$ whenever $g(x)=F(x, y)$ and $g(y)=F(y, x)$. 
Definition 1.6. Let $(X, d)$ be a metric space with $w$-distance $p$. A mapping $F: X \times X \rightarrow X$ is said to be $w$-continuous at a point $(x, y) \in X \times X$ with respect to mapping $g: X \rightarrow X$ if for every $\varepsilon>0$ there exists a $\delta(\varepsilon)>0$ such that $p(g u, g x)+p(g v, g y)<\delta$ implies that $p(F(x, y), F(u, v))<\varepsilon$ for all $u, v \in X$.

Definition 1.7. Let $X$ be a partially ordered set. Mapping $g: X \rightarrow X$ is called strictly monotone increasing mapping if

$$
x \preccurlyeq y \Longleftrightarrow g x \preccurlyeq g y \text { or equivalently } x \succcurlyeq y \Longleftrightarrow g x \succcurlyeq g y \text {. }
$$

Definition 1.8. Let $X$ be a partially ordered set. A mapping $F: X \times X \rightarrow X$ is said to be a mixed monotone if $F(x, y)$ is monotone nondecreasing in $x$ and monotone nonincreasing in $y$, that is, for any $x, y \in X$,

$$
\begin{aligned}
& x_{1}, x_{2} \in X, \quad x_{1} \preccurlyeq x_{2} \Longrightarrow F\left(x_{1}, y\right) \preccurlyeq F\left(x_{2}, y\right), \\
& y_{1}, y_{2} \in X, \quad y_{1} \preccurlyeq y_{2} \Longrightarrow F\left(x, y_{1}\right) \succcurlyeq F\left(x, y_{2}\right) .
\end{aligned}
$$

Kada et al. [1] gave an example to show that $p$ is not symmetric in general. We denote by $M(X)$ and $M_{1}(X)$, respectively, the class of all $w$-distances on $X$ and the class of all $w$ distances on $X$ which are symmetric for comparable elements in $X$. Also in the sequel, we will consider that $(x, y)$ and $(u, v)$ are comparable with respect to ordering in $X \times X$ if $x \succcurlyeq u$ and $y \preccurlyeq v$.

\section{Coupled Coincidence Point}

In this section, we prove coincidence point results in the frame work of partially ordered metric spaces in terms of a $w$-distance.

Theorem 2.1. Let $(X, d)$ be a partially ordered metric space with a w-distance $p$ and $g: X \rightarrow X a$ strictly monotone increasing mapping. Suppose that a mixed monotone mapping $F: X \times X \rightarrow X$ is w-continuous with respect to $g$ such that

$$
p(F(x, y), F(u, v)) \leq a_{1} p(g u, g x)+a_{2} p(g v, g y),
$$

for all $x, y, u, v \in X$ with $x \succcurlyeq u, y \preccurlyeq v$ or $x \preccurlyeq u, y \succcurlyeq v$ and $a_{1}+a_{2}<1$. Let $F(X \times X) \subseteq g(X)$ and $p(y, x)=0$ whenever $p(x, y)=0$, for some $x, y \in \mathrm{cl}_{\omega}(F(X \times X))$. If $g(X)$ is complete and there exist $x_{0}, y_{0} \in X$ such that $g x_{0} \preccurlyeq F\left(x_{0}, y_{0}\right)$ and $F\left(y_{0}, x_{0}\right) \preccurlyeq g y_{0}$, then $F$ and $g$ have a coupled coincidence point.

Proof. Let $g x_{1}=F\left(x_{0}, y_{0}\right)$ and $g y_{1}=F\left(y_{0}, x_{0}\right)$ for some $x_{1}, y_{1} \in X$; this can be done since $F(X \times X) \subseteq g(X)$. Following the same arguments, we obtain $g x_{2}=F\left(x_{1}, y_{1}\right)$ and $g y_{2}=$ $F\left(y_{1}, x_{1}\right)$. Put

$$
\begin{aligned}
& F^{1}\left(x_{0}, y_{0}\right)=g x_{1}, \quad F^{2}\left(x_{0}, y_{0}\right)=F\left(x_{1}, y_{1}\right)=g x_{2} \\
& F^{2}\left(y_{0}, x_{0}\right)=F\left(y_{1}, x_{1}\right)=g y_{2} .
\end{aligned}
$$


Similarly for all $n \in N$,

$$
g x_{n+1}=F^{n+1}\left(x_{0}, y_{0}\right), \quad g y_{n+1}=F^{n+1}\left(y_{0}, x_{0}\right) .
$$

Since $g$ is strictly monotone increasing and $F$ has the mixed monotone property, we have

$$
g x_{2}=F^{2}\left(x_{0}, y_{0}\right)=F\left(x_{1}, y_{1}\right) \succcurlyeq F\left(x_{0}, y_{0}\right)=g x_{1}, \quad g y_{2} \preccurlyeq g y_{1}
$$

Similarly

$$
\begin{aligned}
g x_{0} & \preccurlyeq F\left(x_{0}, y_{0}\right)=g x_{1} \preccurlyeq F^{2}\left(x_{0}, y_{0}\right)=g x_{2} \preccurlyeq \cdots \\
& \preccurlyeq F^{n+1}\left(x_{0}, y_{0}\right)=g x_{n+1} \preccurlyeq \cdots, \\
g y_{0} & \succcurlyeq F\left(y_{0}, x_{0}\right)=g y_{1} \succeq F^{2}\left(y_{0}, x_{0}\right)=g y_{2} \succcurlyeq \cdots \\
& \succcurlyeq F^{n+1}\left(y_{0}, x_{0}\right) \succcurlyeq \cdots
\end{aligned}
$$

Now for all $n \geq 2$, using (2.1), we get

$$
\begin{aligned}
& p\left(F^{n}\left(x_{0}, y_{0}\right), F^{n+1}\left(x_{0}, y_{0}\right)\right) \\
& \quad=p\left(F\left(x_{n-1}, y_{n-1}\right), F\left(x_{n}, y_{n}\right)\right) \\
& \quad \leq a_{1} p\left(g x_{n}, g x_{n-1}\right)+a_{2} p\left(g y_{n}, g y_{n-1}\right) \\
& \quad=a_{1}\left[p\left(F^{n}\left(x_{0}, y_{0}\right), F^{n-1}\left(x_{0}, y_{0}\right)\right)\right]+a_{2}\left[p\left(F^{n}\left(y_{0}, x_{0}\right), F^{n-1}\left(y_{0}, x_{0}\right)\right)\right], \\
& p\left(F^{n}\left(y_{0}, x_{0}\right), F^{n+1}\left(y_{0}, x_{0}\right)\right) \\
& \quad \leq a_{1}\left[p\left(F^{n}\left(y_{0}, x_{0}\right), F^{n-1}\left(y_{0}, x_{0}\right)\right)\right]+a_{2}\left[p\left(F^{n}\left(x_{0}, y_{0}\right), F^{n-1}\left(x_{0}, y_{0}\right)\right)\right] .
\end{aligned}
$$

From (2.6),

$$
\begin{aligned}
& p\left(F^{n}\left(x_{0}, y_{0}\right), F^{n+1}\left(x_{0}, y_{0}\right)\right)+p\left(F^{n}\left(y_{0}, x_{0}\right), F^{n+1}\left(y_{0}, x_{0}\right)\right) \\
& \quad \leq h\left[p\left(F^{n}\left(x_{0}, y_{0}\right), F^{n-1}\left(x_{0}, y_{0}\right)\right)+p\left(F^{n}\left(y_{0}, x_{0}\right), F^{n-1}\left(y_{0}, x_{0}\right)\right)\right],
\end{aligned}
$$

where $h=a_{1}+a_{2}$. Continuing, we conclude that

$$
\begin{gathered}
p\left(F^{n}\left(x_{0}, y_{0}\right), F^{n+1}\left(x_{0}, y_{0}\right)\right)+p\left(F^{n}\left(y_{0}, x_{0}\right), F^{n+1}\left(y_{0}, x_{0}\right)\right) \\
\left.\leq h^{n}\left(p\left(g x_{1}, g x_{0}\right)\right)+p\left(g y_{1}, g y_{0}\right)\right)=h^{n} \delta_{1}
\end{gathered}
$$


if $n$ is odd, where $\delta_{1}=p\left(g x_{1}, g x_{0}\right)+p\left(g y_{1}, g y_{0}\right)$. Also,

$$
\begin{gathered}
p\left(F^{n}\left(x_{0}, y_{0}\right), F^{n+1}\left(x_{0}, y_{0}\right)\right)+p\left(F^{n}\left(y_{0}, x_{0}\right), F^{n+1}\left(y_{0}, x_{0}\right)\right) \\
\leq h^{n}\left(p\left(g x_{0}, g x_{1}\right)+p\left(g y_{0}, g y_{1}\right)\right)=h^{n} \delta_{2}
\end{gathered}
$$

if $n$ is even, where

$$
\delta_{2}=p\left(g x_{0}, g x_{1}\right)+p\left(g y_{0}, g y_{1}\right)
$$

Let $\delta_{n}=p\left(F^{n}\left(x_{0}, y_{0}\right), F^{n+1}\left(x_{0}, y_{0}\right)\right)+p\left(F^{n}\left(y_{0}, x_{0}\right), F^{n+1}\left(y_{0}, x_{0}\right)\right)$; then for every $n$ in $N$ we have

$$
\delta_{n} \leq h^{n} \delta_{0}
$$

where

$$
\delta_{0}=\max \left\{\delta_{1}, \delta_{2}\right\} .
$$

Hence,

$$
p\left(F^{n}\left(x_{0}, y_{0}\right), F^{n+1}\left(x_{0}, y_{0}\right)\right) \longrightarrow 0, \quad p\left(F^{n}\left(y_{0}, x_{0}\right), F^{n+1}\left(y_{0}, x_{0}\right)\right) \longrightarrow 0 \quad \text { as } n \longrightarrow \infty
$$

For $m>n$, we get

$$
\begin{aligned}
& p\left(F^{n}\left(x_{0}, y_{0}\right), F^{m}\left(x_{0}, y_{0}\right)\right)+p\left(F^{n}\left(y_{0}, x_{0}\right), F^{m}\left(y_{0}, x_{0}\right)\right) \\
& \leq p\left(F^{n}\left(x_{0}, y_{0}\right), F^{n+1}\left(x_{0}, y_{0}\right)\right)+p\left(F^{n+1}\left(x_{0}, y_{0}\right), F^{n+2}\left(x_{0}, y_{0}\right)\right)+\cdots \\
&+p\left(F^{m-1}\left(x_{0}, y_{0}\right), F^{m}\left(x_{0}, y_{0}\right)\right) \\
&+p\left(F^{n}\left(y_{0}, x_{0}\right), F^{n+1}\left(y_{0}, x_{0}\right)\right)+p\left(F^{n+1}\left(y_{0}, x_{0}\right), F^{n+2}\left(y_{0}, x_{0}\right)\right)+\cdots \\
&+p\left(F^{m-1}\left(y_{0}, x_{0}\right), F^{m}\left(y_{0}, x_{0}\right)\right) \\
&= \delta_{n}+\delta_{n+1}+\cdots+\delta_{m-1} \leq h^{n} \delta_{0}+h^{n+1} \delta_{0}+\cdots+h^{m-1} \delta_{0} \leq \frac{h^{n}}{1-h} \delta_{0}
\end{aligned}
$$

which further implies that

$$
\begin{aligned}
& p\left(F^{n}\left(x_{0}, y_{0}\right), F^{m}\left(x_{0}, y_{0}\right)\right) \leq \frac{h^{n}}{1-h} \delta_{0} \\
& p\left(F^{n}\left(y_{0}, x_{0}\right), F^{m}\left(y_{0}, x_{0}\right)\right) \leq \frac{h^{n}}{1-h} \delta_{0} .
\end{aligned}
$$


Lemma 1.3(3) implies that $\left\{F^{n}\left(x_{0}, y_{0}\right)\right\}=\left\{g x_{n}\right\}$ and $\left\{F^{n}\left(y_{0}, x_{0}\right)\right\}=\left\{g y_{n}\right\}$ are Cauchy sequences in $g(X)$. Since $g(X)$ is complete, there exist $x, y \in X$ such that $g x_{n} \rightarrow g x$ and $g y_{n} \rightarrow g y$. Since $p\left(g x_{n}, \cdot\right)$ is lower semicontinuous, we have

$$
\left.p\left(F^{n}\left(x_{0}, y_{0}\right)\right), g x\right) \leq \liminf _{m \rightarrow \infty} p\left(g x_{n}, g x_{m}\right) \leq \frac{h^{n}}{1-h} \delta_{0}
$$

which implies that

$$
\left.p\left(F^{n}\left(x_{0}, y_{0}\right)\right), g x\right) \longrightarrow 0 \text { as } n \longrightarrow \infty
$$

Similarly

$$
\left.p\left(F^{n}\left(y_{0}, x_{0}\right)\right), g y\right) \longrightarrow 0 \text { as } n \longrightarrow \infty
$$

Let $\varepsilon>0$ be given. Since $F$ is $w$-continuous at $(x, y)$ with respect to $g$, there exists $\delta>0$ such that for each $n$

$$
p\left(g x_{n}, g x\right)+p\left(g y_{n}, g y\right)<\delta \text { implies that } p\left(F(x, y), F\left(x_{n}, y_{n}\right)\right)<\frac{\varepsilon}{2} .
$$

Since $p\left(g x_{n}, g x\right) \rightarrow 0$ and $p\left(g y_{n}, g y\right) \rightarrow 0$, for $\gamma=\min (\varepsilon / 2, \delta / 2)$, there exists $n_{0}$ such that, for all $n \geq n_{0}$,

$$
p\left(g x_{n}, g x\right)<\gamma, \quad p\left(g y_{n}, g y\right)<\gamma
$$

Now,

$$
\begin{aligned}
p(F(x, y), g x) & \leq p\left(F(x, y), F^{n_{0}+1}\left(x_{0}, y_{0}\right)\right)+p\left(F^{n_{0}+1}\left(x_{0}, y_{0}\right), g x\right) \\
& =p\left(F(x, y), F\left(x_{n_{0}}, y_{n_{0}}\right)\right)+p\left(g x_{n_{0}+1}, g x\right) \\
& <\frac{\varepsilon}{2}+r=\varepsilon
\end{aligned}
$$

implies that $p(F(x, y), g x)=0$. Since

$$
\begin{aligned}
p\left(F^{n}\left(x_{0}, y_{0}\right), F(x, y)\right) & \leq p\left(F^{n}\left(x_{0}, y_{0}\right), g x\right)+p(g x, F(x, y)) \\
& \leq \frac{h^{n}}{1-h} \delta_{0},
\end{aligned}
$$

using Lemma 1.3(1), we obtain $F(x, y)=g x$. Similarly, we can prove that $F(y, x)=g y$. Hence $(x, y)$ is coupled coincidence point of $F$ and $g$. 
Theorem 2.2. Let $(X, d)$ be a partially ordered metric space with a w-distance $p$ having the following properties.

(1) If $\left\{x_{n}\right\}$ is in $X$ with $x_{n} \preccurlyeq x_{n+1}$ for all $n$ and $x_{n} \rightarrow x$ for some $x \in X$, then $x_{n} \preccurlyeq x$ for all $n$.

(2) If $\left\{y_{n}\right\}$ is in $X$ with $y_{n+1} \preccurlyeq y_{n}$ for all $n$ and $y_{n} \rightarrow y$ for some $y \in X$, then $y \preccurlyeq y_{n}$ for all $n$.

Let $F: X \times X \rightarrow X$ be a mixed monotone and $g: X \rightarrow X$ a strict monotone increasing mapping such that

$$
p(F(x, y), F(u, v)) \leq a_{1} p(g u, g x)+a_{2} p(g v, g y),
$$

for all $x, y, u, v \in X$ with $x \succcurlyeq u, y \preccurlyeq v$ or $x \preccurlyeq u, y \succcurlyeq v$ and $a_{1}+a_{2}<1$. Let $F(X \times X) \subseteq g(X)$ and $p(y, x)=0$ whenever $p(x, y)=0$, for some $x, y \in \mathrm{cl}_{\omega}(F(X \times X))$. If $g(X)$ is complete and there exist $x_{0}, y_{0} \in X$ such that $g x_{0} \preccurlyeq F\left(x_{0}, y_{0}\right)$ and $F\left(y_{0}, x_{0}\right) \preccurlyeq g y_{0}$, then $F$ and $g$ have a coupled coincidence point.

Proof. Construct two sequences $\left\{g x_{n}\right\}=\left\{F^{n}\left(x_{0}, y_{0}\right)\right\}$ and $\left\{g y_{n}\right\}=\left\{F^{n}\left(y_{0}, x_{0}\right)\right\}$ such that $g x_{n} \preccurlyeq g x_{n+1}$ and $g y_{n} \succcurlyeq g y_{n+1}$ for all $n$ and $g x_{n} \rightarrow g x$ and $g y_{n} \rightarrow g y$ for some $x \in X$, as given in the proof of Theorem 2.1. Now, we need to show that $F(x, y)=g x$ and $F(y, x)=g y$. Let $\varepsilon>0$. Since $p\left(F^{n}\left(x_{0}, y_{0}\right), g x\right) \rightarrow 0$ and $p\left(F^{n}\left(y_{0}, x_{0}\right), g y\right) \rightarrow 0$, there exists $n_{1} \in N$ such that, for all $n \geq n_{1}$, we have

$$
p\left(F^{n}\left(x_{0}, y_{0}\right), g x\right)<\frac{\varepsilon}{3}, \quad p\left(F^{n}\left(y_{0}, x_{0}\right), g y\right)<\frac{\varepsilon}{3}
$$

\section{Consider}

$$
\begin{aligned}
p(F(x, y), g x) & \leq p\left(F(x, y), F^{n+1}\left(x_{0}, y_{0}\right)\right)+p\left(F^{n+1}\left(x_{0}, y_{0}\right), g x\right) \\
& =p\left(F(x, y), F\left(x_{n}, y_{n}\right)\right)+p\left(F^{n+1}\left(x_{0}, y_{0}\right), g x\right) \\
& \leq a_{1} p\left(g x_{n}, g x\right)+a_{2} p\left(g y_{n}, g y\right)+p\left(F^{n+1}\left(x_{0}, y_{0}\right), g x\right) \\
& =a_{1} p\left(F^{n}\left(x_{0}, y_{0}\right), g x\right)+a_{2} p\left(F^{n}\left(y_{0}, x_{0}\right), g y\right)+p\left(F^{n+1}\left(x_{0}, y_{0}\right), g x\right) \\
& <a_{1} \frac{\varepsilon}{3}+a_{2} \frac{\varepsilon}{3}+\frac{\varepsilon}{3} \\
& <\varepsilon,
\end{aligned}
$$

which implies that $p(F(x, y), g x)=0$. Also, from Theorem 2.1, we have

$$
p\left(F^{n}\left(x_{0}, y_{0}\right), g x\right) \leq \frac{h^{n}}{1-h} \delta_{0}
$$


Therefore,

$$
\begin{aligned}
& p\left(F^{n}\left(x_{0}, y_{0}\right), F(x, y)\right) \\
& \quad \leq p\left(F^{n}\left(x_{0}, y_{0}\right), g x\right)+p(g x, F(x, y)) \\
& \quad \leq \frac{h^{n}}{1-h} \delta_{0}
\end{aligned}
$$

implies that $g x=F(x, y)$. Similarly, we can prove that $F(y, x)=g y$. Hence $(x, y)$ is coupled coincidence point of $F$ and $g$.

\section{Coupled Common Fixed Point}

In this section, using the concept of $w$-compatible maps, we obtain a unique coupled common fixed point of two mappings.

Theorem 3.1. Let all the hypotheses of Theorem 2.1 (resp., Theorem 2.2) hold with $a_{1}+a_{2}<1 / 2$. If for every $(x, y),\left(x^{*}, y^{*}\right) \in X \times X$ there exists $(u, v) \in X \times X$ that is comparable to $(x, y)$ and $\left(x^{*}, y^{*}\right)$ with respect to ordering in $X \times X$, then there exists a unique coupled point of coincidence of $F$ and $g$. Moreover if $F$ and $g$ are $w$-compatible, then $F$ and $g$ have a unique coupled common fixed point.

Proof. Let $\left(g x^{*}, g y^{*}\right)$ be another coupled coincidence point of $F$ and $g$. We will discuss the following two cases.

Case 1. If $(x, y)$ is comparable to $\left(x^{*}, y^{*}\right)$ with respect to ordering in $X \times X$, then

$$
\begin{aligned}
p\left(g x, g x^{*}\right)+p\left(g y, g y^{*}\right) \\
\quad=p\left(F(x, y), F\left(x^{*}, y^{*}\right)\right)+p\left(F(y, x), F\left(y^{*}, x^{*}\right)\right) \\
\quad \leq a_{1} p\left(g x^{*}, g x\right)+a_{2} p\left(g y^{*}, g y\right)+a_{1} p\left(g y^{*}, g y\right)+a_{2} p\left(g x^{*}, g x\right) \\
\quad \leq\left(a_{1}+a_{2}\right)\left[p\left(g x, g x^{*}\right)+p\left(g y, g y^{*}\right)\right]
\end{aligned}
$$

implies that $p\left(g x, g x^{*}\right)+p\left(g y, g y^{*}\right)=0$. Hence $p\left(g x, g x^{*}\right)=0=p\left(g y, g y^{*}\right)$. Also,

$$
\begin{aligned}
p(g x, g x)+p(g y, g y) & =p(F(x, x), F(x, x))+p(F(y, y), F(y, y)) \\
& \leq 2 a_{1} p(g x, g x)+2 a_{2} p(g y, g y)
\end{aligned}
$$

gives that $p(g x, g x)=0=p(g y, g y)$. The result follows using Lemma 1.3(1).

Case 2. If $(x, y)$ is not comparable to $\left(x^{*}, y^{*}\right)$, then there exists an upper bound or lower bound $(u, v)$ of $(x, y),\left(x^{*}, y^{*}\right)$. Again since $g$ is strictly monotone increasing mapping and $F$ satisfies mixed monotone property, therefore, for all $n=0,1, \ldots,\left(F^{n}(u, v), F^{n}(v, u)\right)$ is 
comparable to $\left(F^{n}(x, y), F^{n}(y, x)\right)=(g x, g y)$ and $\left(F^{n}(y, x), F^{n}(x, y)\right)=(g y, g x)$. Following similar arguments to those given in the proof of Theorem 2.1, we obtain

$$
\begin{aligned}
p\left(g x, g x^{*}\right)+p\left(g y, g y^{*}\right)= & p\left(F^{n}(x, y), F^{n}\left(x^{*}, y^{*}\right)\right)+p\left(F^{n}(y, x), F^{n}\left(y^{*}, x^{*}\right)\right) \\
\leq & {\left[p\left(F^{n}(x, y), F^{n}(u, v)\right)+p\left(F^{n}(u, v), F^{n}\left(x^{*}, y^{*}\right)\right)\right] } \\
& +\left[p\left(F^{n}(y, x), F^{n}(v, u)\right)+p\left(F^{n}(v, u), F^{n}\left(y^{*}, x^{*}\right)\right)\right] \\
= & {\left[p\left(F^{n}(x, y), F^{n}(u, v)\right)+p\left(F^{n}(y, x), F^{n}(v, u)\right)\right] } \\
& +\left[p\left(F^{n}(u, v), F^{n}\left(x^{*}, y^{*}\right)\right)+p\left(F^{n}(v, u), F^{n}\left(y^{*}, x^{*}\right)\right)\right] \\
\leq & h^{n} \beta_{0}+h^{n} \gamma_{0},
\end{aligned}
$$

where $\beta_{0}=\max \{p(g u, g x)+p(g v, g y), p(g x, g u)+p(g y, g v)\}$ and $\gamma_{0}=\max \left\{p\left(g x^{*}, g u\right)+\right.$ $\left.p\left(g y^{*}, g v\right), p\left(g u, g x^{*}\right)+p\left(g v, g y^{*}\right)\right\}$. On taking limit as $n \rightarrow \infty$ on both sides of (3.3), we have

$$
p\left(g x, g x^{*}\right)+p\left(g y, g y^{*}\right)=0
$$

and $p\left(g x, g x^{*}\right)=0=p\left(g y, g y^{*}\right)$. By the same lines as in Case 1, we prove that $p(g x, g x)=$ $0=p(g y, g y)$. Again Lemma 1.3(1) implies that $g x=g x^{*}$ and $g y=g y^{*}$. Hence $(g x, g y)$ is unique coupled point of coincidence of $F$ and $g$. Note that if $(g x, g y)$ is a coupled point of coincidence of $F$ and $g$, then $(g y, g x)$ are also a coupled points of coincidence of $F$ and $g$. Then $g x=g y$ and therefore $(g x, g x)$ is unique coupled point of coincidence of $F$ and $g$. Let $u=g x$. Since $F$ and $g$ are $w$-compatible, we obtain

$$
g u=g(g x)=g(F(x, x))=F(g x, g x)=F(u, u) .
$$

Consequently $g u=g x$. Therefore $u=g u=F(u, u)$. Hence $(u, u)$ is a coupled common fixed point of $F$ and $g$.

Remark 3.2. If in addition to the hypothesis of Theorem 2.1 (resp., Theorem 2.2) we suppose that $p \in M_{1}(X), x_{0}$ and $y_{0}$ are comparable, then $g x=g y$.

Proof. Recall that $g x_{0} \preccurlyeq F\left(x_{0}, y_{0}\right)$. Now, if $x_{0} \preccurlyeq y_{0}$, then $g x_{0} \preccurlyeq g y_{0}$. We claim that, for all $n \in N, g x_{n} \preccurlyeq g y_{n}$. Since $g$ is strictly monotone increasing and $F$ satisfies mixed monotone property, we have

$$
g x_{1}=F\left(x_{0}, y_{0}\right) \preccurlyeq F\left(y_{0}, x_{0}\right)=g y_{1}
$$

Assuming that $g x_{n} \preccurlyeq g y_{n}$, since $g$ is strictly monotone increasing, so $x_{n} \preccurlyeq y_{n}$. By the mixed monotone property of $F$, we have

$$
g x_{n+1}=F^{n+1}\left(x_{0}, y_{0}\right)=F\left(x_{n}, y_{n}\right) \preccurlyeq F\left(y_{n}, x_{n}\right)=g y_{n+1} .
$$


Therefore,

$$
g x_{n} \preccurlyeq g y_{n} \quad \forall n .
$$

Letting $\varepsilon>0$, there exists an $n_{0} \in N$ such that $p\left(g x, F^{n}\left(x_{0}, y_{0}\right)\right)<\varepsilon / 4$ and $p\left(F^{n}\left(y_{0}, x_{0}\right), g y\right)<$ $\varepsilon / 4$ for all $n \geq n_{0}$. Now,

$$
\begin{aligned}
p(g x, g y) & \leq p\left(g x, F^{n_{0}+1}\left(x_{0}, y_{0}\right)\right)+\left(F^{n_{0}+1}\left(x_{0}, y_{0}\right), g y\right) \\
& \leq p\left(g x, F^{n_{0}+1}\left(x_{0}, y_{0}\right)\right)+p\left(F^{n_{0}+1}\left(x_{0}, y_{0}\right), F^{n_{0}+1}\left(y_{0}, x_{0}\right)\right)+\left(F^{n_{0}+1}\left(y_{0}, x_{0}\right), g y\right) \\
& <\frac{\varepsilon}{4}+h p\left(F^{n_{0}}\left(x_{0}, y_{0}\right), F^{n_{0}}\left(y_{0}, x_{0}\right)\right)+\frac{\varepsilon}{4} \\
& \leq \frac{\varepsilon}{2}+h\left[p\left(F^{n_{0}}\left(x_{0}, y_{0}\right), g x\right)+p(g x, g y)+\left(g y, F^{n_{0}}\left(y_{0}, x_{0}\right)\right)\right] \\
& <\frac{\varepsilon}{2}+h \frac{\varepsilon}{4}+h p(g x, g y)+h \frac{\varepsilon}{4} \\
& <\varepsilon+h p(g x, g y)
\end{aligned}
$$

implies that $(1-h) p(g x, g y)<\varepsilon$. Since $h<1$, therefore $p(g x, g y)=0$. Similarly we can prove that $p(g x, g x)=0$. Hence by Lemma 1.3(1), we have $g x=g y$. Similarly, if $g x_{0} \succcurlyeq g y_{0}$, we can show that $g x_{n} \succcurlyeq g y_{n}$ for each $n$ and $g x=g y$.

\section{Acknowledgment}

The present version of the paper owes much to the precise and kind remarks of the learned referees.

\section{References}

[1] O. Kada, T. Suzuki, and W. Takahashi, "Nonconvex minimization theorems and fixed point theorems in complete metric spaces," Mathematica Japonica, vol. 44, no. 2, pp. 381-391, 1996.

[2] D. Ilić and V. Rakočević, "Common fixed points for maps on metric space with w-distance," Applied Mathematics and Computation, vol. 199, no. 2, pp. 599-610, 2008.

[3] L. Guran, "Fixed points for multivalued operators with respect to a w-distance on metric spaces," Carpathian Journal of Mathematics, vol. 23, no. 1-2, pp. 89-92, 2007.

[4] L.-J. Lin and W.-S. Du, "Some equivalent formulations of the generalized Ekeland's variational principle and their applications," Nonlinear Analysis: Theory, Methods E Applications, vol. 67, no. 1, pp. 187-199, 2007.

[5] L.-J. Lin and W.-S. Du, "Systems of equilibrium problems with applications to new variants of Ekeland's variational principle, fixed point theorems and parametric optimization problems," Journal of Global Optimization, vol. 40, no. 4, pp. 663-677, 2008.

[6] J. R. Morales, "Generalizations of Some Fixed Point Theorems," Notas de mathematica, 1999. Pre-Print, no. 199.

[7] J. R. Morales, "Fixed point's theorems for $\omega$ - $\varphi$-contractions," Notas de mathematica, 2004. Pre-Print, no. 230. 
[8] Q. H. Ansari, "Vectorial form of Ekeland-type variational principle with applications to vector equilibrium problems and fixed point theory," Journal of Mathematical Analysis and Applications, vol. 334 , no. 1, pp. 561-575, 2007.

[9] J.-S. Ume, "Fixed point theorems related to Cirić's contraction principle," Journal of Mathematical Analysis and Applications, vol. 225, no. 2, pp. 630-640, 1998.

[10] W. Takahashi, Nonlinear Functional Analysis: Fixed Point Theory and Its Applications, Yokohama Publishers, Yokohama, Japan, 2000.

[11] T. G. Bhaskar and V. Lakshmikantham, "Fixed point theorems in partially ordered metric spaces and applications," Nonlinear Analysis: Theory, Methods and Applications, vol. 65, no. 7, pp. 1379-1393, 2006.

[12] F. Sabetghadam, H. P. Masiha, and A. H. Sanatpour, "Some coupled fixed point theorems in cone metric spaces," Fixed Point Theory and Applications, vol. 2009, Article ID 125426, 8 pages, 2009.

[13] V. Lakshmikantham and L. Ćirić, "Coupled fixed point theorems for nonlinear contractions in partially ordered metric spaces," Nonlinear Analysis: Theory, Methods \& Applications, vol. 70, no. 12, pp. 4341-4349, 2009. 